Monika Schumacher*

\title{
C2+-DaF-Lernende reflektieren über eine perfekte Sprachbeherrschung - ist diese vor dem Hintergrund der postulierten funktionalen Mehrsprachigkeit in Alltag und Beruf künftig noch gefragt?
}

DOI 10.1515/cercles-2015-0015

Abstract: By adopting "functional plurilingualism" - a recurring concept in its new curriculum, "Lehrplan 21" - Switzerland is pursuing new avenues in foreign language education. The term refers to a goal-oriented approach to foreign language learning and teaching in primary and secondary schools, and implies that barriers to communication can be overcome by exploiting the sum of the individual's linguistic capacities. In future, language learners will be expected to build on the interrelatedness of languages instead of developing abilities in languages that they keep separate from one another. This view of foreign language education coincides with the recommendations of the Council of Europe's Common Framework of Reference for Languages (CEFR). Currently, however, instruction follows more traditional paths; generally speaking, languages continue to be learned one at a time.

Having reached the highest level specified by the CEFR, some learners continue their studies, seeking to maintain or reinforce their competences or to address individual weaknesses. In this article, a group of $\mathrm{C} 2+$ learners of German as a foreign language reflect on their desire for linguistic perfection and the notion of "functional plurilingualism" against the background of their own biographies. These learners have a high awareness and knowledge of German (often their third language) and of the learning process, which allows them to make informative statements. The data was analysed qualitatively and subjective theories were reconstructed - an approach that took account both of the multiperspectivity of the topic and of the uniqueness of the individual cases.

Key words: functional plurilingualism, native-like proficiency, German as a foreign language, learning biography, identity

*Corresponding author: Monika Schumacher, Dr.phil., Zürcher Hochschule für Angewandte Wissenschaften, Language Competence Centre, Deutsch als Fremdsprache, E-mail: scok@zhaw.ch 


\section{Einleitung}

In einem modernen Europa soll die kulturelle und sprachliche Vielfalt als gemeinsames Erbe betrachtet und entsprechend gehegt und gepflegt werden. In der Gesellschaft wie im individuellen Falle bisher oft als Hindernis stigmatisiert, könnte Mehrsprachigkeit so fortan eine Quelle der gegenseitigen Bereicherung sein und als Ressource dienen. Dieser Prozess ist nicht zu bewältigen, ohne dass das Bildungs- und Erziehungswesen eine Leitrolle übernimmt (vgl. Council of Europe 1982).

Dem Begriff der «funktionalen Mehrsprachigkeit» bin ich zum ersten Mal in der Fachdidaktik an der Pädagogischen Hochschule begegnet. In dem im Zusammenhang mit der Schulharmonisierung in der Schweiz entwickelten und sich zurzeit in der Vernehmlassung befindenden «Lehrplan 21» heisst es im Bereich «Sprachen»:

Die funktionale Mehrsprachigkeit bezeichnet die Fähigkeit eines Menschen, unter schiedliche Sprachen und Sprachformen bzw. Register erfolgreich, der Situation und dem Gegenüber angepasst zu nutzen (D-EDK 2013: 2).

Aus dem erwähnten Dokument geht die zentrale Rolle des Konzepts deutlich hervor. Dass die Schülerinnen und Schüler eine funktionale Mehrsprachigkeit bis zum Ende der offiziellen Schulzeit erreichen, ist ein erklärtes Ziel und zugleich ein aktueller Ansatz im Umgang mit dem Fremdsprachenerwerb in der Staatsschule.

Drei Orte der eigenen Lehrtätigkeit haben mich zur Reflexion über das Konzept angeregt. Wie die Mehrsprachigkeit allgemein im aktuellen Diskurs definiert wird, darauf gehe ich im Folgenden noch ein. Zunächst werden aber besagte Orte mittels einer jeweils kurzen Anekdote vorgestellt:

In der Fachdidaktik Englisch an einer pädagogischen Hochschule sollten die Studierenden als Studienleistung eine Unterrichtssequenz filmen. Als Dozierende evaluierte ich die Arbeiten anschliessend. Eine Studentin gab eine sehr überzeugende Arbeit $a b$, die ich gern als Beispiel für andere Klassen bzw. Jahrgänge aufbewahrt hätte. $\mathrm{Zu}$ diesem Zweck jedoch wollte mir die Studentin eine Kopie ihrer Arbeit nicht überlassen. Die Studierenden seien ausgesprochen kritisch und sie wolle sich nicht lächerlich machen, ihr Englisch sei akzent- und fehlerbehaftet. Die Pädagogische Hochschule bildet angehende Lehrende aus. Sie werden ihren Unterricht auf einen Lehrplan ausrichten, der sich für den Bereich «Sprachen» der «funktionalen Mehrsprachigkeit» verschrieben hat. Aus der Aussage der Studierenden liesse sich schliessen, dass das Konzept (noch) nicht verinnerlicht worden ist. Mit 
welcher Einstellung gegenüber der Sprache werden die Junglehrerinnen und Junglehrer Kinder unterrichten?

Szenenwechsel: In der Justizvollzugsanstalt Pöschwies lernen die fremdsprachigen Insassen auf freiwilliger Basis Deutsch. In der täglichen Kommunikation mit den Betreuenden sind sie meist auf sämtliche kommunikativen Mittel angewiesen, über die sie, aber auch die Kommunikationspartner, verfügen. Funktionale Mehrsprachigkeit kann im engen Korsett einer totalen Institution (vgl. Goffman 1961), wo reibungslose Abläufe zentral sind, vieles erleichtern. Daneben gäbe und gibt es aber noch andere Möglichkeiten, Teilkompetenzen zu nutzen, so etwa die rezeptive Mehrsprachigkeit. Entscheidend für die Wahl einer Teilkompetenz sind letzten Endes die Kommunikationspartner, Kommunikationszweck und -ort (vgl. Haider 2010: 207-208).

Und schliesslich der dritte Ort: eine Gruppe Deutschlernender an der Zürcher Hochschule für Angewandte Wissenschaften (ZHAW). Die Kursteilnehmenden haben entweder das Niveau C2 erreicht und das Zertifikat der Stufe erlangt, oder sie haben mittels eines Einstufungstests ihre exzellenten Deutschkenntnisse nachgewiesen. Die Motivation der Lernenden, sich weiter zu verbessern, ist hoch. Die deutsche Sprache wird dabei isoliert betrachtet. Es geht um ihre Finessen, um höchstmögliche Präzision und letzten Endes um Perfektion. Die Arbeit mit dem gegenseitigen Ansporn innerhalb der Gruppe ist für die Lernenden und für mich als Kursleiterin hochmotivierend.

In diesem Artikel steht Deutsch als Fremdsprache im Zentrum, ich werde daher nicht weiter auf den fremdsprachlichen Unterricht an den öffentlichen Schulen bzw. die Ausbildung an der Pädagogischen Hochschule eingehen. Auch der zweite oben genannte Kontext wird in der folgenden Diskussion ausgeblendet. Ich verweise für eine eingehendere Auseinandersetzung damit auf meine Dissertation (vgl. Schumacher 2012). Der Frage nach der Rolle der «funktionalen Mehrsprachigkeit» bei Lernenden mit nahezu perfekten Deutschkenntnissen möchte ich an dieser Stelle mein ausschliessliches Augenmerk schenken. Hier siedelte ich eine kleine empirische Untersuchung an, mit deren Ergebnissen ich meine Ausführungen in diesem Artikel unterfüttern werde. Zunächst aber möchte ich das Konzept der Mehrsprachigkeit im europäischen Kontext definieren.

\section{Auf dem Weg zu einem mehrsprachigen Europa}

Der Gemeinsame europäische Referenzrahmen für Sprachen hält zur Mehrsprachigkeit fest: 
[Sie] ... betont die Tatsache, dass sich die Spracherfahrung eines Menschen in seinen kulturellen Kontexten erweitert, von der Sprache im Elternhaus über die Sprache der ganzen Gesellschaft bis zu den Sprachen anderer Völker [...]. Diese Sprachen und Kulturen werden aber nicht in strikt voneinander getrennten mentalen Bereichen gespeichert, sondern bilden vielmehr gemeinsam eine kommunikative Kompetenz, zu der alle Sprachkenntnisse und Spracherfahrungen beitragen und in der die Sprachen miteinander in Beziehung stehen und interagieren (Europarat 2001: 17).

Bei der Mehrsprachigkeit geht es also um die Koexistenz verschiedener Sprachen und Kulturen in einer Gesellschaft. Damit unterscheidet sie sich von der Vielsprachigkeit, der Kenntnis einer Anzahl von Sprachen, die durch ein vielfältiges Sprachangebot an Schulen und in Bildungssystemen und durch die Dominanz der englischen Sprache auf dem internationalen Parkett erzielt werde. Unausgewogenheit sei bei der Mehrsprachigkeit natürlich, denn die mehrsprachige und plurikulturelle Kompetenz verfüge über ein kurzlebiges Profil und eine veränderliche Konfiguration. Durch Veränderungen und Erfahrungen in Beruf, Familie und Freizeit sowie beim Reisen werde die linguistische und kulturelle Biographie eines Individuums geprägt und verändert. Durch die Komplexität an Erfahrungen entstehe im Idealfall ein besseres Bewusstsein der Identität (Europarat 2001: 133).

Zwar verwendet der Gemeinsame europäische Referenzrahmen für Sprachen den kombinierten Begriff «funktionale Mehrsprachigkeit» an keiner Stelle, nennt aber in der Definition der Mehrsprachigkeit die Möglichkeit des einzelnen Sprechers, der einzelnen Sprecherin, in verschiedenen Situationen flexibel auf verschiedene Teile der Kompetenz zurückzugreifen, um eine effektive Kommunikation mit einem bestimmten Gesprächspartner oder einer -partnerin zu erreichen. Damit nimmt er meiner Ansicht nach zumindest implizit auf das im «Lehrplan 21» explizit benannte Bezug.

Flexibel wird etwa dann auf Teilkompetenzen zugegriffen, wenn eine oder beide Parteien in einem Gespräch von einer Sprache oder einem Dialekt zu einer oder einem anderen wechseln und dadurch alle Möglichkeiten der jeweiligen Sprache oder Varietät ausreizen. Weiter wird die Möglichkeit genannt, sich in einer Sprache auszudrücken und den Partner oder die Partnerin in der anderen bloss $\mathrm{zu}$ verstehen. Hilfreich seien auch der Einsatz von Internationalismen, möglicherweise verfügbaren Sprachmittlern/Sprachmittlerinnen oder von allem linguistischen Wissen - auch paralinguistische Mittel wie Mimik, Gestik, Gesichtsausdruck (sic!).

Es liegt auf der Hand, dass, wenn Sprachen nicht mehr isoliert gelernt werden, sich ein völlig neuer Ansatz des Sprachunterrichts ergibt. Wie aber soll ein solcher Paradigmen-wechsel vonstatten gehen? Die Bildungsinstitutionen sollen 
sicherstellen, dass das Ziel der kommunikativen, mehrsprachlichen Kompetenz, ein «sprachliches Repertoire» also, erreicht werden kann. Doch Lernen findet auch - und besonders - ausserhalb des Schulkontextes statt:

[W]e learn new facts, skills, ideas and emotional capacities simply by virtue of enrolling with that permanently instructive institution, the University of Life [...]. In this broad meaning of the term, you cannot stop yourself from being a lifelong learner. It covers pretty much everything - and rightly so. (Field 2000: vii.-viii.)

Im Sinnes eines Lebenslangen Lernens - Lifelong Learning (vgl. Faure et al. 1972 und das 2000 in Lissabon von der Kommission der europäischen Gemeinschaften verabschiedete «Memorandum on Lifelong Learning») sollen und müssen Spracherfahrungen auch ausserhalb der Schule bzw. im späteren Leben gemacht werden können, dazu muss die Motivation der Lernenden erhalten bleiben, müssen ihre Fähigkeiten und ihr Selbstvertrauen gestärkt werden. Meines Erachtens findet weiterführendes Lernen mit dem Wunsch nach Kompetenzerweiterung bereits im (klassischen) Lernen von einzelnen Sprachen statt. Wie wird es sich ausgestalten, wenn die Lernenden ihr sprachübergreifendes Repertoire einsetzen?

\section{Eine nahezu muttersprachliche Beherrschung der Fremdsprache als Kontrast zum Konzept der «funktionalen Mehrsprachigkeit»?}

Ich habe in der Einleitung drei Kontexte genannt, in denen ich mir als Fremdsprachenlehrende Gedanken zur «funktionalen Mehrsprachigkeit» machen konnte. Wie bereits dort erwähnt, nehme ich in der folgenden Diskussion auf die Gruppe der C2+-Lernenden im Deutsch als Fremdsprachenunterricht Bezug. Gemäss der Kann-Beschreibung im Gemeinsamen europäischen Referenzrahmen für Sprachen «können sich [Lernende, die das Niveau C2 erreicht haben] mühelos an allen Gesprächen und Diskussionen beteiligen». Das bedeutet mit anderen Worten, dass auf einem C2Niveau monolingual sämtliche kommunikativen Momente problemlos bewältigt werden können, darum auch der Ausdruck «mastery», um die C2-Stufe mittels eines Begriffs zu definieren. Selbstverständlich sind diese Aussagen auf eine theoretische bzw. ideale und nicht konkrete Kommunikation bezogen. Es ist hinlänglich bekannt, dass Kommunikation in der Praxis sehr komplex und 
daher störanfällig ist (vgl. Bohnsack $1992 \mathrm{zu}$ den verschiedenen Theorien der Kommunikationsanalyse). Die Gruppe der hier vorgestellten Lernenden verfügt über nahezu muttersprachliche Kenntnisse der deutschen Sprache. Kommt es hier zu kommunikativen Missverständnissen, könnten diese sogar ein Beweis der sprachlichen Perfektion sein, in dem Sinne, dass das Gegenüber aus sprachlichen Gründen nicht zurückstecken muss, sondern aufgrund der quasi-muttersprachlichen Kompetenzen des Gesprächspartners/der Gesprächspartnerin eine symmetrische Ausgangslage annimmt. Wenn also die Studierenden keine sprachlichen Hürden mehr $\mathrm{zu}$ überwinden haben oder nur solche, wie sie jeder Kommunikationskonstellation inhärent sein können, warum lernen sie dann nach dem höchsten zu erreichenden Niveau auch weiterhin Deutsch? Ist der Wunsch nach maximaler Sprachbeherrschung nicht ein Widerspruch zur postulierten funktionalen Mehrsprachigkeit in Alltag und Beruf. Sollen zeitliche und finanzielle Ressourcen tatsächlich dafür eingesetzt werden?

Natürlich könnte man diesen Überlegungen entgegenhalten, dass die von mir Befragten allesamt in der Deutschschweiz leben und auch in Zukunft hier leben werden. Sie haben sich im Zielland der Fremdsprache niedergelassen. Wenn wir aber von einem zukünftigen funktionalen Ansatz der Mehrsprachigkeit für Europa ausgehen, dann wäre die Frage nach dem aktuellen Lebensraum unerheblich, da Fremdsprachen dereinst nur noch vernetzt und nicht mehr isoliert voneinander gelernt würden und dies bereits von Kindsbeinen an.

Den Wunsch nach perfekter Beherrschung einer Fremdsprache vor dem Hintergrund einer postulierten «funktionalen Mehrsprachigkeit» und weitere damit verbundene Fragen untersuchte ich durch ein empirisches Vorgehen. Die Studie wird im Folgenden erörtert.

\section{Die empirische Studie}

Im Rahmen meiner Befragung habe ich mit neun $\mathrm{C} 2+$-Lernenden extensive halbstrukturierte Interviews von einer Länge von je etwa 90 Minuten geführt. Im Zentrum stand die individuelle Lernbiographie der Einzelnen.

\subsection{Der Leitfaden zur Befragung}

Der Leitfaden orientierte sich an zentralen Themen:

- Die erste(n) Fremdsprache(n)

- Die erste Begegnung mit der deutschen Sprache 
- Die individuellen Erfahrungen beim Sprachenlernen: Höhenflüge, Stolpersteine, die eigene Wahrnehmung der Sprache

- Die Verwendung der deutschen Sprache im Deutschschweizer bzw. deutschen Kontext

- Gründe, das Lernen nach dem Erreichen der Stufe C2 fortzusetzen

- Der Begriff der «funktionalen Mehrsprachigkeit» in der eigenen Lernbiographie

- Eine Selbsteinschätzung der eigenen, aktuellen sprachlichen Situation.

\subsection{Das Sample}

Das Sample bestand aus neun Lernenden, wobei nur die Daten von sieben für die Auswertung hinzugezogen wurden. Die Daten von zwei Lernenden wurden nicht ausgewertet. In einem Fall handelt es sich um eine Lernende, die in der italienischsprachigen Schweiz aufgewachsen ist, im anderen Fall um das einzige männliche Sample, das jedoch das Niveau C2 noch nicht erreicht hat. Diese Entscheidung sollte einer von der sprachlichen Kompetenz aus betrachtet homogeneren Datenmenge dienen. Die folgende Tabelle führt die für die Befragung

\section{Natascha $30+$ Russland Deutsch, Englisch Primarlehrerin}

4. Tereza 20+ Tschechische Republik Deutsch, Englisch, Spanisch Studentin angehenden Primarlehrerin

\section{Stefania $50+$}

Spanien

Deutsch,

Französisch

Kunsthistorikerin

Spanischlehrerin

5. Matilde $40+$

Portugal

Deutsch,

Französisch,

Englisch, Spanisch

Übersetzerin,

Spanischlehrerin

7. Zsófia $60+$

Ungarn

Englisch, Russisch, Französisch

Russisch- u. Englischlehrerin

3. Hiromi $50+$ Japan Deutsch, Englisch, Französisch, Spanisch Bankangestellte

\section{Judy $40+$}

Schottland

Deutsch, Französisch,

Spanisch

Steuerberaterin,

Studentin - Übersetzen

Darstellung 1: Das Sample - eine Auswahl. 
relevanten soziobiografischen Variablen der Probandinnen auf. Zum Sample ist noch zu sagen, dass die Tatsache, dass kein einziger Lerner dabei ist, durchaus repräsentativ für diese Stufe ist. Hingegen ist es eher ein Zufall, dass für die Zeit der Befragung vier von sieben Probandinnen von Berufs wegen mit Sprachen zu tun haben.

\subsection{Subjektive Theorien als Auswertungsinstrument}

Mit einem empirischen Vorgehen wollte ich die Sichtweise der Befragten ins Zentrum des Interesses rücken und dabei besonders die Einzelfälle hervorheben. Das Forschungsprogramm FST für «Forschungsprogramm Subjektive Theorien» (Groeben und Scheele 1977), das Ende der 70er Jahre des letzten Jahrhunderts entstand, und seither kontinuierlich weiterentwickelt wurde, gilt als der theoretisch und methodisch am detailliertesten ausgearbeitete Ansatz hierfür (Steinke 1999: 53). Ich habe das entsprechende qualitative Verfahren in der Empirie zu meiner Dissertation eingesetzt und gute Erfahrungen damit gemacht (vgl. Schumacher 2012).

Für das Konzept werden folgende Merkmale definiert:

- Kognitionen der Selbst- und Weltsicht

- ein komplexes Aggregat mit (zumindest impliziter) Argumentationsstruktur, das auch die zu objektiven (wissenschaftlichen) Theorien parallelen Funktionen der Erklärung, Prognose, Technologie erfüllt (Scheele und Groeben 1988: 16).

Subjektive Theorien enthalten subjektive Konstrukte, subjektive Daten und subjektive Hypothesen oder auch Gesetzmässigkeiten (Groeben et al. 1988: 54ff.). Analog zu objektiven Theorien werden auch hier Bewertungskriterien formuliert. Das Kriterium der Validität, der Gültigkeit der Annahmen, hat seine Entsprechung in der Veridikalität, d.h. der Glaubwürdigkeit (Groeben et al. 1988: 107ff.).

Der Inhalt von Subjektiven Theorien wird zunächst mittels qualitativer Verfahren erhoben. Dann werden durch die Struktur-Lege-Technik so genannte Strukturbilder gelegt. Anschliessend wird die ermittelte subjektive Theorie im Dialog-Konsens kommunikativ und dann explanativ validiert. Ist sie realitätsadäquat? In welchem Ausmass kann sie handlungsleitend sein? Aus methodologischer und forschungspraktischer Sicht wird das Verfahren kritisch betrachtet: Es stellt sich die Frage nach der Asymmetrie zwischen der forschenden und der befragten Person, bei der Validierung scheint es fragwürdig, eine Subjektive Theorie 
auf objektive Richtigkeit hin zu prüfen. Zudem ist die Interrelation von Kognition und Handeln bisher nicht eindeutig geklärt (vgl. Bovet 1993: 15-23 und 15-16; Grotjahn 1993; Kallenbach 1995, 1996; Steinke 1999: 6).

Im für das FST zentralen Element Dialog-Konsens stehen Befragende und Befragte auf hierarchisch gleicher Stufe. Damit wird von einem epistemologischen Menschenbild ausgegangen: Der Mensch ist ein handelndes Subjekt, das über Intentionalität, Reflexivität, potenzielle Rationalität und sprachliche Kommunikationsfähigkeit verfügt (Grotjahn 1998: 38).

Caspari (2003: 106-107) schlägt für die Analyse der Interviewdaten ein mehrstufiges Verfahren zur Rekonstruktion der Subjektiven Theorie vor, dem ich weitgehend gefolgt bin:

1. Eine vollständige und wörtliche Transkription aller Interviews (die Transkriptionen der Interviews werden hier nicht abgedruckt, können aber bei der Autorin eingesehen werden).

2. Die Analyse und Interpretation aller Aussagen, die $\mathrm{zu}$ einem der unter 4.1 aufgelisteten Leitfadenthemen gehörten und das Formulieren von Grobkategorien daraus.

3. Das Verdichten der Ergebnisse aus den Grobkategorien zu «Statements» (zentrale Aussagen der Befragten) und das Entwickeln von unterschiedlich gewichteten Feinkategorien für jede einzelne Probandin auf der Basis der Grobkategorien.

4. Das Arrangieren der Statements zu einem Strukturbild mit Vernetzungen zwischen den einzelnen Aussagen und einer erkennbaren Gesamtstruktur.

Auf den letzten Schritt der Deutungsmusteranalyse und Gesamtinterpretation wurde verzichtet, da es an dieser Stelle nicht um eine weitere Verdichtung der Strukturbilder ging. Solche Formen der Modifikation der einzelnen Phasen werden von Groeben et al. (1988) eingeräumt.

\subsubsection{Grobkategorien aus den Subjektiven Theorien als Diskussionsgrundlage}

Aus dem Leitfaden der Interviews und der Analyse der Sequenzen haben sich Grobkategorien ableiten lassen. Sie sind hier als Frage oder Wortgruppe formuliert. Von mir weiter ausformuliert werden sie als Ausgangspunkt der unter Punkt 4 folgenden Diskussion eingesetzt:

1. Fremdspracherwerb hauptsächlich zu kommunikativen Zwecken?

2. Flüssigkeit versus Korrektheit

3. Die Bedeutung von verschiedenen Sprachen im globalen Wettbewerb

4. Affekt versus Emotion 
5. Funktionale Ziele versus perfekte Sprachbeherrschung

6. Kontinuierlicher Wissens- bzw. Kompetenzzuwachs?

\section{Wie nehmen die Probandinnen Aspekte der Mehrsprachigkeit wahr?}

Bei der folgenden Präsentation der Daten setze ich als Untertitel jeweils das Statement einer Probandin ein, das mir für die Diskussion als aussagekräftig erscheint. Die Statements der Probandinnen dienen weiter dem Vorantreiben der Diskussion. Sie wurden in Form und Sprache nicht angepasst, lediglich die Pausen und Interjektionen wie «äh» oder «ähm» wurden nicht abgetippt.

\section{1 «Bei Bernhard Schlinks, Vorleser’ habe ich es zum ersten Mal als eine schöne Sprache gesehen»}

Mit der «funktionalen Mehrsprachigkeit» werden die kommunikativen Kompetenzen der Lernenden ins Zentrum des Fokus gerückt; das KommunizierenKönnen ist der hauptsächliche Zweck des Mehrspracherwerbs. Verlieren bei einem solchen Ansatz rezeptive Kompetenzen ganz allgemein ihre Relevanz? Welche Rolle könnte dem Lesen und dem Hören von Texten zukommen?

Für Probandin 7 liegt klar auf der Hand, dass sie durch sämtliches Gedrucktes bzw. Geschriebenes beim Deutschlernen sehr profitieren konnte und immer noch profitiert - v.a. auch indem sie sich dabei auf eine Metaebene begibt: «Bis heute lese ich alles, was ich sehe und was geschrieben ist und das hilft viel: Zeitungen, Bücher, ich schmökere in einer Buchhandlung. Man entdeckt viel» (Zsófia).

Probandin 1 reflektiert ebenfalls über die deutsche Sprache, deren hohe Präzision sie sehr schätzt, gerade deshalb will sie nicht auf andere Sprachen zurückgreifen: «Deutsch ist so reizvoll, weil es so strukturiert und präzise ist, dass man so viele Ereignisse und Vorkommnisse so klar und präzise definieren kann wie in keiner anderen Sprache» (Natascha).

Probandin 6 streicht besonders den ästhetischen Wert des Gedruckten hervor, den sie erst auf einem sehr hohen sprachlichen Niveau voll auskosten kann; für sie mit einer eher negativen Haltung gegenüber der Schönheit der deutschen Sprache eine Art Schlüsselerlebnis.

Bei Bernhard Schlinks «Vorleser» habe ich es zum ersten Mal als eine schöne Sprache gesehen. Ich hatte es schon auf Englisch gelesen, auf Deutsch ist es aber so viel schöner. 
Das hat mich überrascht, ich hatte nicht erwartet, dass ich etwas auf Deutsch schöner als auf Englisch finden könnte. (Judy)

In der folgenden Aussage von Probandin 3 wird deutlich, dass das sehr gute Beherrschen der Zielsprache bei einem sozialen Anlass sehr relevant sein kann und dass diesbezüglich unter den fremdsprachigen Anwesenden eine - vielleicht unausgesprochene bzw. sehr subjektiv wahrgenommene - Konkurrenz besteht. Das sprichwörtliche Funktionieren in der Kommunikation ist hier sozial erwünscht, allerdings nur, wenn die Sprecherin dabei monolingual agiert.

Wir sind oft an Anlässen eingeladen. Dort sind dann acht, zehn Leute an einem Tisch, es wird geplaudert und ich muss rasch reagieren können. In Gesellschaft bin ich oft die einzige Asiatin, die meisten Fremdsprachigen haben schon in der Schule sehr gut Deutsch gelernt. Es ist brutal. (Hiromi)

Die Probandinnen gehen in ihren Aussagen stark von der Charakteristik des Deutschen aus. Sie finden darin den Reiz der Sprache. Die Sprache ist nicht ausschliesslich Mittel zur Kommunikation, sie befassen sich mit ihr ebenfalls auf einer Metaebene. Man könnte sogar sagen, dass für sie die Relevanz der gewählten Sprache und ihre Charakteristika über dem In-der-Sprache-kommunizieren-Können steht.

\section{2 «Wenn man sehr schlecht spricht oder gebrochen, das stelle ich mir so vor, wie wenn man auf Pflaster stolpert»}

Wenn der Fokus beim Fremdspracherwerb auf die Flüssigkeit in der kommunikativen Äusserung gelegt wird, verliert dann die Korrektheit bzw. ein akkurater Umgang mit der Fremdsprache an Bedeutung? Unser Schulsystem ist stark sprachlastig. Ohne die hiesige Sprache zu beherrschen bzw. mit mangelhaften Kenntnissen, kann man die öffentliche Schule nicht durchlaufen, ganz zu schweigen von einer höheren Bildung. Das Messen von Sprachkompetenz dient ausserdem der Selektion. Ist es möglich, in der Muttersprache einen Ansatz zu wählen, der auf Korrektheit basiert und dann aber ganz anders in der Fremdsprache die Flüssigkeit ins Zentrum zu stellen? Wie können im frühen Stadium des Fremdsprachenerwerbs Kompetenzen im sprachlich-systematischen Bereich erworben und aufgebaut und v.a. wie können Einbussen in diesem Bereich verhindert werden? 
Probandin 2 unterrichtet Kunstgeschichte und dies auf Deutsch. Sie kann sich ausdrücken, die Schüler können ihrem Unterricht folgen. Dennoch ist ihr das zu wenig.

Seit drei Jahren unterrichte ich wieder Kunstgeschichte. Mir sind aber meine vielen Fehler bewusst. Ich möchte mich korrekter ausdrücken. Wenn man sehr schlecht spricht oder gebrochen, das stelle ich mir so vor, wie wenn man auf Pflaster stolpert. Ständig stolpert. Man denkt, man ist ja dumm. (Stefania)

Probandin 7 weist darauf hin, dass die Mehrsprachigkeit und damit das Interagieren von mehreren Sprachen bei einer lernenden Person ebenso zu Interferenzen führen kann, wie der Vergleich mit der Muttersprache: «Ich habe entdeckt, dass die ,language interference' nicht nur zwischen Muttersprache und Fremdsprache stattfindet, sondern auch zwischen zwei Fremdsprachen» (Zsófia). Dieser Umstand wäre doch eigentlich ein Argument für den fundierten Erwerb einer isolierten Fremdsprache.

Problematisch erscheint es, wenn eingeschränkte Sprachkenntnisse dazu führen, dass die Zuhörenden Rückschlüsse auf die Muttersprache des Sprechenden ziehen: «Wie man eine Fremdsprache lernt, zeigt auch, wie man die Muttersprache beherrscht. Spricht jemand fehlerhaft, denke ich, das hat eine Verbindung zur Person selber» (Hiromi).

Man könnte die folgende Aussage von Probandin 4 mit dem Phänomen «ethnolektales Deutsch» (vgl. Hinnenkamp 2010: 71) vergleichen: Man kann Sprachregeln nur brechen, wenn man die Sprache sehr gut beherrscht. Sehr schön kommt hier die Freude an der Sprache Deutsch zum Ausdruck:

Unter Freunden oder mit meinem Mann gebrauche ich manchmal neuen Wortschatz in einer übertriebenen Form. Das kam, weil ich Gehobensprachliches in die Umgangssprache einbauen wollte. Die anderen machen dann auch Vorschläge, das ist manchmal lustig. (Tereza)

\section{3 «Der grösste Stolperstein war für mich, dass jemand sagte, meine Sprache ist komisch»}

Meiner Meinung nach wird die Rolle des Gegenübers bzw. der Gesellschaft überhaupt mit dem Ansatz der «funktionalen Mehrsprachigkeit» weitgehend vernachlässigt. Es ist schön und wünschenswert, dass das Individuum mit seiner Mehrsprachigkeit und seiner anderen Weltansicht mehr Bedeutung und dadurch auch mehr Wertschätzung erfährt. Über die Aufnahme und Wahrnehmung des Versuchs zu kommunizieren, i.e. die Rezeption beim Adressaten, wird jedoch 
wenig gesagt. Doch Kommunikation bedingt ja immer ein Gegenüber, sie ist grundsätzlich eher asymmetrisch, was vor allem mit der Machtverteilung zu tun hat.

Der französische Soziologe Pierre Bourdieu bringt mit dem Konzept «linguistisches Kapital» zum Ausdruck, dass Sprache und Macht eng verquickt sind. Auf dem Sprachmarkt, existiere eine einheitliche Währung, nämlich die legitime Sprache, womit die allgemein anerkannten Vorstellungen von einer «richtigen» Sprache gemeint sind. Das Sprachkapital jedoch sei ungleich verteilt, denn für die Verteilung seien diejenigen Systeme und Institutionen verantwortlich, die zugleich die Macht inne hätten. Die soziale Position des Einzelnen ermögliche oder verhindere den Zugang zu einer Sprache. Die Sprache wiederum könne Bildung erschweren oder verunmöglichen. Damit werde die Sprache zur Währung und Teil eines kulturellen Kapitals. Wer Sprachkapital habe, sei sprachkompetent, d.h. fähig zur Produktion, Aneignung und Bewertung von Sprache (Bourdieu 1990: 46). Der Markt bzw. die gesellschaftlichen Akteure und Institutionen bestimmten, was anerkannter Gebrauch und somit korrekt sei. Sprachlicher Habitus sei die untrennbar technische und soziale Kompetenz, zu sprechen und auf eine bestimmte sozial ausgezeichnete Weise zu sprechen. Treffe dieser mit dem Markt zusammen, entstehe als Produkt Diskurs (Bourdieu 1989: 42). Im Kleinen beginnt der Markt bereits in der Kindheit zu greifen, so haben Wieman und Giles (1992) aufgezeigt, dass, weil der soziale Status eines Kindes das Ergebnis sozialer Interaktionen ist, die Ausdrucksfähigkeit in der Sprache, die in der Schulklasse gesprochen wird, eine grosse Rolle für die Akzeptanz und den Einfluss spielt.

Von diesen Überlegungen auf die konkrete Kommunikationskonstellation schliessend, muss man sich im Zusammenhang mit Macht die Frage stellen, ob Kommunikation nicht per se eine asymmetrische Ausgangslange schafft. Des Weiteren gilt es $\mathrm{zu}$ berücksichtigen, wie der Kommunikationspartner auf den Versuch der Kommunikation bzw. auf das Kommunizieren selber eingeht.

Probandin 1 macht mit ihrer Aussage dazu den hohen Druck deutlich, der beim Kommunizieren auf ihr lastet, wenn sie etwas mit Nachdruck sagen möchte: «Ein Gefühl der Unterlegenheit kommt immer wieder vor, wenn ich aufgeregt bin, etwas begründen will, meine Meinung durchsetzten will» (Natascha).

Die folgende Bemerkung von Probandin 2 zeigt, dass die Akzeptanz der ganzen Person durch das Umfeld eingeschränkt ist, wenn die Sprache den Erwartungen nicht voll entspricht.

Auch Kollegen haben sich negativ verhalten. Einer meinte, meine Korrektur sei nicht präzise. «Wir sind hier in der Schweiz, da wird so etwas ernst genommen. - Vielleicht 
ist es in Spanien anders.» - Ein Experte bei der Maturitätsprüfung. Ich habe mich beschwert, er hatte keinen Grund, so etwas zu sagen. (Stefania)

Was von der Gesellschaft als sprachlich inadäquat betrachtet und kritisiert wird, betrifft Probandin 4 in ihrer Persönlichkeit sehr direkt. «Der grösste Stolperstein war für mich, dass jemand sagte, meine Sprache ist komisch. Das tut dann sehr weh, das kann am Anfang vieles kaputt machen» (Tereza).

Dass, wer die hiesige Sprache nicht voll beherrscht, nicht oder nur begrenzt vertrauenswürdig sein kann, erfuhr Probandin 2:

Als mein Sohn ganz klein war, wurde er krank. Der Arzt verschrieb ein Antibiotikum. Es war kurz vor Ladenschluss. Ich ging in die Apotheke und mir fehlte ein wenig Kleingeld. Man wollte mir das Medikament nicht geben. Es war die Apotheke in der Nähe unserer Wohnung und ich hatte schon mehrmals dort etwas gekauft. Ich musste ein langes Formular ausfüllen, fast einen Schwur ablegen. Ganz wichtig war der Name meines Mannes. (Stefania)

Dass es aber nicht unbedingt die Sprache in erster Linie ist, die zu negativen Äusserungen führt, erlebte Probandin 5: «Ich treffe immer noch auf Vorurteile. Ich habe diese Woche einer älteren Frau im Bus meinen Platz angeboten. Sie fragte mich, ob ich Deutsch verstehe. Hat vielleicht mit meinem Äusseren zu tun» (Matilde).

Probandin 7 wusste die negativen Einstellungen des Umfeldes gegenüber ihren Sprachkenntnissen in positive Energie umzusetzen. Sie bekam so den Ansporn, sich auf die GDS-Prüfung vorzubereiten: «Die Motivation für das GDS war, meinen Schülern zu beweisen, dass ich auch Hochdeutsch kann. Ich habe oft arrogantes Verhalten von Gymnasiasten erfahren, bloss weil ich mich vielleicht nicht fliessend ausgedrückt habe» (Zsófia).

\section{4 «Deutsch als Liebessprache. Ein ganz anderer Bezug»}

Wie unter 4. festgehalten soll der einzelne Sprecher/die einzelne Sprecherin und seine/ihre sprachlichen Kompetenzen durch die funktionale Mehrsprachigkeit aufgewertet werden. Wir kommunizieren, die Kommunikation funktioniert alles bestens also. Aber wo bleiben bei dieser Sichtweise die Emotionen? Können wir gefühlvoll funktionieren oder funktional Gefühle zeigen? Da scheint der Begriff an seine Grenzen zu kommen, denn ihm haftet etwas zu Technisches an. So gesehen bekommt der Einzelne/die Einzelne durch den Ansatz nicht einen höheren Stellenwert, vielmehr findet eine Verarmung der Kommunikation statt; das Individuum und seine Bedürfnisse gehen im Funktionieren unter.

Die Sprache ist ja gleichzeitig Werkzeug zur Erzeugung und Ausdruckmittel affektiver Zustände. Im Unterricht erleben die Lernenden solche Zustände, die 
jedoch schwer in der Fremdsprache auszudrücken seien. Erschwerend komme hinzu, dass Lernziele vernachlässigt würden, die zum Ausdruck von Emotionen befähigen (vgl. Iluk 2002: 97), vielmehr verfolgt man «verkopfte» Lernwege und -ziele. Wenn sich die Lernenden der eigenen Emotionen bewusst würden und diese in der Fremdsprache auch artikulieren könnten, gelänge es, den Lernprozess vereinfachende Emotionen zu fördern (Arnold 1999: 2).

Wir alle kennen die Erfahrung, dass wir etwas, zu dem wir einen emotionalen Bezug haben, leichter bzw. schneller lernen. Der Wunsch, eine Fremdsprache $\mathrm{zu}$ lernen, steht vielleicht im direkten Zusammenhang mit einem anderen Menschen. Probandin 5 hat es so erlebt und schildert dies folgendermassen: «Entscheidend war, dass ich mich verliebt habe, Deutsch als Liebessprache. Ein ganz anderer Bezug» (Matilde). Idealerweise ist diese Liebe eine nachhaltige; dazu noch einmal Probandin 5: «Je mehr man in die Kultur hineingeht, umso mehr gewinnt auch die Sprache an Schönheit» (Matilde).

Wie beeinflussen Affekt und Emotionen der Lernenden die Funktionalität? Wenn die Funktionalität der Sprache im Vordergrund steht, kann diese dann immer noch in ihrer Ästhetik wahrgenommen und dafür geschätzt, bevorzugt und geliebt werden? Ist die funktionale Kommunikation zwingend ein prosaisches In-Kontakt-Treten? Hier zeigt sich meiner Meinung nach das Paradoxe des Begriffs «Funktionale Mehrsprachigkeit»: Wer kommunizieren kann, ist funktional. Aber braucht es nicht gerade in der Kommunikation - also beim Funktionieren - das Emotionale. Hierzu äussert sich Probandin 2: «Emotionen sind die letzte Stufe. Das Beispiel ist meine Mutter, die, wenn sie zärtlich war, nur Katalanisch gesprochen hat. Ich könnte nie dem Sohn und dem Mann gegenüber so viele Emotionen zeigen wie in der Muttersprache» (Stefania).

Ist nicht oft der Grund der Kommunikation ein emotionaler, man möchte Gefühle ausdrücken? Probandin 3 erkennt ihre sprachlichen Unzulänglichkeiten, wenn sie Emotionales äussern soll: «Ich komme bei sehr formellen Briefen und bei Emotionen an die Grenzen. Klar hat man Gestik und Mimik. Es ist mehr der genau passende Ausdruck» (Hiromi).

Ein weiterer Aspekt, der von Probandin 6 aufgeworfen wurde und hier zumindest kurz gestreift werden soll, ist der Humor: «Ich habe oft das Gefühl, dass ich eine andere Person bin, wenn ich Deutsch spreche, oder dass ich nicht ich bin. Humor lässt sich teilweise nicht übersetzen» (Judy).

\section{5 «Eine rein funktionale Sprache - das ,funktioniert’ nicht»}

Die Lernenden konzentrieren sich auf eine einzige Sprache. Selbstverständlich stellen sie beim Lernen Querbezüge zur Muttersprache oder zu einer anderen 
Fremdsprache her. Aber in erster Linie geht es ihnen um ein möglichst perfektes Beherrschen der Fremdsprache. Wenn man von der Funktionalität ausgeht, wie viel Perfektion innerhalb einer isolierten Sprache ist dann wünschenswert? Stehen die beiden Ansätze nicht in einem dialektischen Verhältnis zueinander? Probandin 4 erwähnt die Vorbildfunktion, die einem als Lehrerin oder Lehrer oft zufällt: «Es gibt immer so eine Person, die du bewunderst von der Sprache, meistens ist es der Lehrer oder die Lehrerin, und du versuchst dich wie anzupassen. Sprechen wie die Person, nachahmen. Das ist dein Vorbild» (Tereza). Darf man sich als Lernende in Zukunft muttersprachliche Sprecherinnen und Sprecher generell noch zum Vorbild nehmen? Wie definieren die Lernenden ihre eigenen Ziele bei einem solchen Ansatz? Solche Gedanken haben Implikationen nicht nur für das Lernen, sondern auch für das Lehren: Wie werden wir Lehrende künftig die Sprachbeherrschung einer einzelnen Sprache beurteilen und messen? Wie definieren wir unsere Pflichten und Aufgaben und unsere Verantwortung gegenüber den Lernenden?

Für die Probandinnen 4, 5 und 7 (Zitate in dieser Reihenfolge) kommt das Verharren in funktional-kommunikativen Kompetenzen einer geistigen Verarmung gleich: «Wenn man zufrieden ist mit ,normal', dann würde es reichen» (Tereza); «Ob die Funktionalität ausreicht, kommt sehr auf die eigenen Ansprüche an. Ich persönlich möchte mehr» (Matilde); «Wenn man höhere intellektuelle Bedürfnisse hat, reicht kommunikatives Funktionieren nicht. Eine rein funktionale Sprache, das wäre etwas Künstliches meiner Ansicht nach, das ,funktioniert' nicht» (Zsófia).

Probandin 2 stellt in Frage, ob sich mit einem funktionalen Ansatz überhaupt berufliche und private Beziehungen entwickeln und aufbauen lassen: «Mit Funktionalität kann man keine Freundschaften schliessen, keine Gefühle ausdrücken, komplizierte berufliche Beziehungen knüpfen, etwas aushandeln, wo es Missverständnisse und Uneinigkeiten geben kann» (Stefania).

Probandin 6 räumt ein, dass man sehr wohl die Sprache als reines Mittel zur kommunikativen Funktion betrachten kann. Allerdings beruft sie sich dabei auf ihre Anfänge in der deutschen Sprache, in denen sie mit viel Widerwillen lernte und ohne sich der Sprache zu öffnen: «Deutsch habe ich am Anfang nur funktional gesehen: logisch mit Regeln» (Judy).

\section{Wohin sind die Probandinnen gelangt? - «lch habe etwas verloren. Aber es ist nicht negativ»}

Mit einem Blick auf den Status quo der einzelnen Befragten möchte ich die Diskussion abschliessen. 
Vorausschauend auf ein Europa der Zukunft implizieren der Text im «Lehrplan 21» und auch die Ausführungen zum Gemeinsamen europäischen Referenzrahmen für Sprachen, dass die Kommunikation mit Anderssprachigen, die Mobilität und die zunehmende Offenheit dem anderen gegenüber zu einem Zuwachs an Kompetenzen und Wissen führt. Gleiches scheint im Streben nach Perfektion ausgeschlossen. Sämtliche Probandinnen haben selbstinitiiert das Gespräch auf dieses für sie mitunter auch schwierige Thema gelenkt. So kommt also hier als abschliessender Punkt der Diskussion die Frage nach dem Erreichten auf. Es ist auffällig, dass bei allen Befragten melancholische Gefühle mitschwingen; sie thematisieren den Gewinn, aber eben auch den (dabei) entstandenen Verlust. Was haben die Lernenden erreicht und welches sind ihre weiteren Ziele?

Probandin 3 betrachtet sich als jemand, der nicht (mehr) über eine dominante Sprache verfügt, gleichzeitig aber in allen Sprachen, die er spricht, Defizite aufweist. «Ich habe etwas verloren. Aber es ist nicht negativ. Ich denke, meine Hauptsprache ist Deutsch. Japanisch ist weit weg. Wenn wir plötzlich ins Englische wechseln, habe ich Mühe. Ich bin nicht bilingual, sondern semilingual geworden» (Hiromi).Probandin 4 erlebt das In-denHintergrund-Treten der eigenen Muttersprache sehr bewusst: «Manchmal bin ich nostalgisch. Ein Abschied auf Raten. Nicht, dass ich das nicht gut finde. Es hat leider beides, das ist manchmal das Schwierige. Auf Tschechisch bin ich manchmal schon unsicher» (Tereza).

Probandin 5 sieht das Problem u.a. in der räumlichen Distanz: «Für ein hohes Niveau in der Fremdsprache muss man etwas von der eigenen Sprache aufgeben. Das tut weh. Aber man muss sich entwickeln. Ich versuche, beide Kulturen zu pflegen. Aber man ist dort nicht auf dem Laufenden» (Matilde). Dieselbe Befragte zeigt aber auch, dass eine gute Integration und eine perfekte Sprachbeherrschung sie teilweise ihren Landsleuten entfremdet hat und immer noch entfremdet:

Bei meinem Einsatz für [eine Hilfsorganisation] ist es ganz schwierig für mich, an meine Landsleute zu kommen. Ich bin für die eine Fremde, vielleicht eine Schweizerin. Ich lebe in einer ganz andern Welt. Es gibt diese Schwelle. Wie kann man sie überwinden? Die Kollegen, die Erfolg haben, können ganz wenig Deutsch. Die sind funktional. (Matilde)

Umgekehrt soll die Befragte 4 im eher einfachen beruflichen Alltag, wo sie als Studentin arbeitet, dem Stereotyp einer tschechischen Deutschlernenden mit Akzent entsprechen: «Ich glaube, die Leute, die in der Bäckerei einkaufen, mögen mich und finden es auch schön, dass ich nicht so perfekt spreche. Sie sagen, ich solle es ein bisschen behalten, auch die Fehler manchmal» (Tereza). 
Vielleicht kokettiert die Befrage 2 nur, wenn sie die perfekte Sprachbeherrschung mit dem Untergehen in der Masse gleichsetzt: «Perfekt integriert zu sein, die Sprache in Perfektion zu beherrschen, eine unter den anderen zu sein - vielleicht möchte man das auch nicht» (Stefania).

Aus den Aussagen der Lernenden geht hervor, dass der Weg zu einem nahezu perfekten Deutsch, in der Form, wie sie es bereits erreicht haben, oder auch in Zukunft weiter anstreben, nicht ohne Abstriche in der eigenen Muttersprache oder einer anderen ersten Fremdsprache abläuft und daher teilweise auch schmerzlich ist. Sie beschäftigen sich tiefgehend mit der Sprache, was Einsatz und Fleiss bedingt. Ein funktionaler Ansatz, wo fundierte Kenntnisse der Fremdsprache gar nicht angestrebt werden, da Grenzen ja durch ein Ausweichen auf andere Strategien und Sprachen umgangen werden können, scheint weniger anstrengend $\mathrm{zu}$ sein. Zudem ist auch die Reibungsfläche geringer, weil das Individuum mit seiner Identität weniger direkt betroffen ist. Spontan erscheint die «funktionalen Mehrsprachigkeit» als die längerfristig effektivere Methode bzw. als zielorientiert, für ein künftiges Europa, wo ein sehr hohes Mass an Mobilität herrscht und die kulturelle und sprachliche Durchmischung in den einzelnen Staaten sehr gross ist. Aber im praktischen Handeln des Alltags muss sich das Konzept erst noch bewähren.

\section{Ein paar abschliessende Bemerkungen}

Ganz bewusst sollte mein Text die Perspektive der Lernenden ausleuchten. Sie sind ja eigentliche Lernexpertinnen und -experten. Korrektheit und Perfektion in einer Fremdsprache anzustreben, scheint einen Sinn zu haben. Das haben die Auszüge aus den Interviews meiner Meinung nach deutlich gezeigt. Eine derart hohe Sprachbeherrschung hat für die Befragten weniger mit elitärem Denken zu tun, als vielmehr mit dem Wunsch, in einem deutschsprachigen Kontext dazuzugehören. Andere Lernende haben andere Zielmarken, je nach individuellen Vorstellungen und Möglichkeiten.

In der Volksschule werden jedoch die Grundlagen für späteres Sprachenlernen gelegt. Es muss im Sinne eines lebenslangen Lernens zwingend nachhaltig sein. Wenn dort die «funktionale Mehrsprachigkeit» postuliert wird, prägt dies einzelne Sprachlernbiografien und betrifft schliesslich auch uns Sprachenlehrende. Wir haben hier die Verantwortung, Entwicklungen kritisch $\mathrm{zu}$ verfolgen. Wie werden wir in Zukunft Sprachen unterrichten? Was werden unsere Ziele sein? - Dies wären weitere Fragen $\mathrm{zu}$ einem aktuellen und ganz offensichtlich sehr komplexen Thema. 


\section{Literaturliste}

Arnold, Jane (Hrsg.). 1999. Affect in language learning. Cambridge: Cambridge University Press. Bohnsack, Ralf. 1992. Interaktion und Kommunikation. In Hermann Korte \& Bernhard Schäfers (Hrsg.), Einführung in die Hauptbegriffe der Soziologie, 35-57. Opladen: Springer.

Bourdieu, Pierre. 1989. Satz und Gegensatz. Über die Verantwortung des Intellektuellen. Berlin: Klaus Wagenbach.

Bourdieu, Pierre. 1990. Was heisst sprechen? Die Ökonomie des sprachlichen Tausches. Wien: Braumüller.

Bovet, Gislinde. 1993. Wie sieht guter Psychologieunterricht aus? Ermittlung und Erörterung der subjektiven didaktischen Theorien von Psychologielehrerinnen und -lehrern über guten, machbaren Psychologieunterricht in der gymnasialen Oberstufe. Frankfurt am Main: Peter Lang.

Caspari, Daniela. 2003. Fremdsprachenlehrerinnen und Fremdsprachenlehrer - Studien zum beruflichen Selbstverständnis. Tübingen: Gunter Narr.

Commission of the European Communities. 2000. A memorandum on lifelong learning. Brussels: European Commission.

Council of Europe. 1982. Recommendation no. R(82) 18 of the Committee of Ministers to member States concerning modern languages. Appendix A to Denis Girard \& John L. M. Trim.1988. Project no. 12: Learning and teaching modern languages for communication: Final report of the project group (activities 1982-1987). Strasbourg: Council of Europe.

D-EDK (Deutschschweizer Erziehungsdirektoren-Konferenz). 2013. Lehrplan 21 - Sprachen. Konsultationsfassung. Abrufbar unter: http://konsultation.lehrplan.ch/downloads/ container/31_1_0_1_1.pdf.

Europarat. 2001. Gemeinsamer europäischer Referenzrahmen für Sprachen: lernen, lehren, beurteilen. Berlin und München: Langenscheidt.

Faure, Edgar, Felipe Herrera, Abdul-Razzak Kaddoura, Henri Lopes, Arthur V. Petrovsky, Majid Rahnema \& Frederik Champion Ward. 1972. Learning to be: The world of education today and tomorrow. UNESCO. Abrufbar unter: http://unesdoc.unesco.org/images/0000/ 000018/001801e.pdf.

Field, John. 2000. Lifelong learning and the new educational order. Stoke on Trent: Trentham Books.

Goffman, Erving. 1961. Asylums: Essays on the social situation of mental patients and other inmates. New York: Doubleday.

Groeben, Norbert \& Brigitte Scheele. 1977. Argumente für eine Psychologie des reflexiven Subjekts. Paradigmawechsel vom behavioralen zum epistemologischen Menschenbild. Darmstadt: Steinkopff.

Groeben, Norbert, Diethelm Wahl, Jörg Schlee \& Brigitte Scheele. 1988. Das Forschungsprogramm Subjektive Theorien: eine Einführung in die Psychologie des reflexiven Subjekts. Tübingen: Francke.

Grotjahn, Rüdiger. 1993. Qualitative vs. quantitative Fremdsprachenforschung: Eine klärungsbedürftige und unfruchtbare Dichotomie. In Johannes-Peter Timm \& Helmut Johannes Vollmer (Hrsg.), Kontroversen in der Fremdsprachenforschung: Dokumentation des 14. Kongresses für Fremdsprachendidaktik, 223-248. Bochum: Brockmeyer.

Grotjahn, Rüdiger 1998. Subjektive Theorien in der Fremdsprachenforschung: Methodologische Grundlagen und Perspektiven. In Gert Henrici \& Ekkehard Zöfgen, Fremd-sprachen Lehren und Lernen, 33-59. Tübingen: Gunter Narr. 
Haider, Barbara. 2010. Übung, die. In Hans Barkowski \& Hans-Jürgen Krumm (Hrsg.), Fachlexikon Deutsch als Fremd- und Zweitsprache, 207-208. Stuttgart: UTB.

Hinnenkamp, Volker. 2010. Ethnolekt, der. In Hans Barkowski \& Hans-Jürgen Krumm (Hrsg.), Fachlexikon Deutsch als Fremd- und Zweitsprache, 71. Stuttgart: UTB.

Iluk, Jan. 2002. Probleme der Befähigung zum Ausdruck von Emotionen in der Fremdsprache aus curricularer Sicht. Deutsch als Fremdsprache 2. 96-103.

Kallenbach, Christiane. 1995. Das Konzept der subjektiven Theorien aus fremdsprachendidaktischer Sicht. In Lothar Bredella \& Herbert Christ. (Hrsg.). Didaktik des Fremdverstehens. 81-96. Tübingen: Narr (= Giessener Beiträge zur Fremdsprachendidaktik).

Kallenbach, Christiane. 1996. Subjektive Theorien. Was Schüler und Schülerinnen über Fremdsprachen denken. Tübingen: Gunter Narr.

Scheele, Brigitte \& Norbert Groeben. 1988. Dialog-Konsens-Methoden zur Rekonstruktion Subjektiver Theorien: die Heidelberger Struktur-Lege-Technik (SLT), konsensuale ZielMittel-Argumentation und kommunikative Flussdiagramm-Beschreibung von Handlungen. Tübingen: Francke.

Schumacher, Monika. 2012. Sprachgebrauch in der Justizvollzugsanstalt Pöschwies - Zum sprachlichen Umgang zwischen fremdsprachigen Gefangenen und den Anstaltsmitarbeitenden im Spannungsfeld von sprachlicher und institutioneller Asymmetrie. Fribourg: Universität Fribourg, E-Thesis. Online abrufbar unter: Sprachgebrauch in der Justizvollzugsanstalt Pöschwies. Working Papers in Applied Linguistics, Departement Angewandte Linguistik, Fachartikel, ZHAW.

Steinke, Ines. 1999. Kriterien qualitativer Forschung. Ansätze zur Bewertung qualitativ-empirischer Sozialforschung. Weinheim: Juventa.

Wiemann, John. M. \& Howard Giles. 1992. Interpersonale Kommunikation. In Geoffrey M. Stephenson (Hrsg.), Sozialpsychologie. Eine Einführung, 209-231. Berlin: Springer.

\section{Bionote}

\section{Monika Schumacher}

Monika Schumacher (Dr. phil.) ist langjährige Dozentin für Deutsch als Fremdsprache auf allen Stufen, u.a. im Justizvollzug. Ihre Arbeitsschwerpunkte sind das Lernen auf einem quasi muttersprachlichen Niveau, ebenso jenes, unter erschwerten Bedingungen. Ihre Forschungsinteressen liegen im soziolinguistischen Bereich. 